\title{
The effect of vacuum freeze-drying and radiation on allogeneic aorta grafts
}

\author{
QING CAO $^{1 *}$, LEREN TAO $^{2 *}$, MENGFANG LIU $^{2}$, MENG YIN $^{3}$ and KUN SUN ${ }^{4}$ \\ ${ }^{1}$ Department of Pediatrics, Shanghai Children's Medical Center Affiliated to Shanghai Jiaotong University, \\ Shanghai 200127; ${ }^{2}$ Institute of Cryogenic Technology and Frozen Foods, Shanghai University of Technology, \\ Shanghai 200093; ${ }^{3}$ Department of Pediatric Cardiothoracic Surgery, Shanghai Children's Medical Center \\ Affiliated to Shanghai Jiaotong University, Shanghai 200127; ${ }^{4}$ Department of Pediatric Cardiology, \\ Xinhua Hospital Affiliated to Shanghai Jiatong University, Shanghai 200092, P.R. China
}

Received May 24, 2012; Accepted October 10, 2012

DOI: $10.3892 / \mathrm{mmr} .2012 .1146$

\begin{abstract}
Vacuum freeze-dried aorta is a satisfactory material for blood vessel grafting. Previous studies have focused on immunity, however, vacuum freeze-drying is a complicated process of heat and mass transfer, and adopting a programmed cooling process may more completely preserve the mechanical properties of the blood vessels. Irradiation, as a method of removing pathogens, lowers the antigenic activity of the blood vessels. In our study, vacuum freeze-drying combined with radiation was used as a treatment for porcine aorta prior to grafting, aimed at deactivating endogenous retrovirus, shielding masses of endothelial cells and lowering the immunogenicity of the blood vessels. As for the mechanical properties, compared with normal aorta, the maximum axial tensile stress (ATS) decreased by $20 \%$, the maximum circumferential tensile stress (CTS) increased by $30 \%$ and the maximum puncture stress (PT) decreased by $20 \%$. Our results revealed that 2 months after of grafting, the host cells had migrated into the graft tissue and propagated to initiate endothelialization, the inflammatory reaction was abated and the PT had returned to normal levels.
\end{abstract}

Correspondence to: Dr Kun Sun, Department of Pediatric Cardiology, Shanghai Jiaotong University Affiliated to Xinhua Hospital, 1665 Kongjiang Road, Shanghai 200092, P.R. China E-mail: caoqingsunkun@126.com

Dr Meng Yin, Department of Pediatric Cardiothoracic Surgery, Shanghai Children's Medical Center Affiliated to Shanghai Jiaotong University, Shanghai 200127, P.R. China

E-mail: ymym12th@163.com

*Contributed equally

Key words: vacuum freeze-drying, radiation, immunogenicity, mechanical properties, porcine endogenous retrovirus

\section{Introduction}

As the numbers of cases of cardiovascular disease steadily grow, grafting as a therapeutic method has been demonstrated to be effective, and millions of tissues and organs are demanded (1). However, as there is a shortage of donor tissues and organs and low graft efficiency, the clinical application of aorta grafting has been restrained (2-4). Since the 1980s, a variety of substitutes have been used for aorta grafting. Artificial materials, biomaterials and composites have been tested as substitutes for blood vessels in practical applications and experiments (5-7). Due to rejection, thrombosis, endothelial cell hyperplasia and calcification, they are not very suitable for blood vessels with a diameter $<1 \mathrm{~cm}(8)$. Thus, at present, substitutes are not able to completely replace freeze-banked human tissues and organs.

Three requirements for grafting are reduced immunogenicity, preserved vessel structure and mechanical properties, and elevated histocompatibility (9-12). Given the variety of fatal diseases that may infect through blood, including AIDS and SARS, the tissue or organ graft should be pathogen-free.

Cryopreservation technologies represent potentially long-term and minimally damaging methods for the preservation of tissues. The conventional cryopreservation of allogeneic blood vessels involving freezing is currently being used clinically, but in vivo studies using these grafts have exhibited poor long-term patency rates due to tissue structure injury and changes in mechanical properties (13). A suitable method of tissue preservation is urgently required.

With highly structural and functional homology, porcine tissues and organs are used as replacements for human ones, which, to some extent, has eased the situation of a deficit of tissues and organs. Due to calcification and high immunogenicity, the clinical application of porcine material has been limited (14). A new method of reducing immunogenicity and elevating histocompatibility is required for porcine xenografts.

The current study introduces a process of vacuum freeze-drying combined with radiation treatment for the pre-grafted aorta, aiming at reducing immunogenicity, preserving vessel structure and mechanical properties, elevating histocompatibility and removing pathogenic microorganisms from the allogenetic aorta graft. The tissue structure, 
mechanical properties, pathogen content and immunogenicity of the graft are measured and discussed.

\section{Materials and methods}

Reagents and instruments. D-Hanks' solution and PBS solution were acquired from Sangon (Shanghai, China). The experimental instruments used were as follows: S450 scanning electron microscope (Hitachi, Tokyo, Japan), optical microscope (Olympus, Tokyo, Japan), texture analyzer (Trapezium Lite, Hong Kong, China), freeze drier (SP Industries, Inc., Warminster, PA, USA) and ABI Prism 7000 real-time PCR system (Beckman-Coulter, Miami, FL, USA).

Animals and sampling. Young pigs weighing 10-15 kg were supplied by a Shanghai pig farm. All animal experiments were carried out in accordance with the guide for Care and Use of Laboratory Animals (the 'NIH Guide'). The protocols for the use of animals were approved by the Department of Laboratory Animal Sciences from Shanghai Jiao Tong University School of Medicine.

Vacuum freeze-drying combined with radiation treatment. The aortas, $\sim 2 \mathrm{~cm}$ in length, were dissected out from the pigs under anesthesia. The isolated aortas were washed with D-Hanks' solution and PBS solution, once each. The aortas were applied to a programmed cooling system as described in our previous study (15). Briefly, the aortas were cooled to $-43^{\circ} \mathrm{C}$ at a cooling rate of $1^{\circ} \mathrm{C}$ per minute. The aortas were positioned vertically and kept at $-43^{\circ} \mathrm{C}$ for $1 \mathrm{~h}$. The aortas were then dried at $-20^{\circ} \mathrm{C}$ for $2 \mathrm{~h}$ and at $15^{\circ} \mathrm{C}$ for $4 \mathrm{~h}$, while the pressure was kept at 3-10 $\mathrm{Pa}$. The aortas were then put into a sealed and sterile apparatus, irradiated with $20 \mathrm{kGy}{ }^{60} \mathrm{Co}$ rays for $8 \mathrm{~h}$ and preserved at room temperature. Prior to performing follow-up experiments, the dried aortas were immersed in physiological saline for $2 \mathrm{~h}$.

Histological observation. The aortas were fixed in $40 \mathrm{~g} / 1$ formaldehyde solution for $48 \mathrm{~h}$. Following dehydration using ethanol and xylene, the samples were embedded in paraffin and sectioned at $5 \mu \mathrm{m}$. Subsequently, hematoxylin and eosin (H\&E) staining was performed.

Immunohistochemistry. The aorta (1 cm in length) was dissected out and then cut into frozen sections at a thickness of $5 \mu \mathrm{m}$. The sections were fixed in formaldehyde solution for 1 min. After washing once with PBS solution, the sections were placed into a humidor. The primary antibody (MHC) at a dilution of 1:100 was added to the sections, and PBS solution replaced the primary antibody in the negative controls. The slides were maintained at $37^{\circ} \mathrm{C}$ for $1 \mathrm{~h}$. After extensive washing with PBS solution, the secondary antibody (FITC-conjugated rabbit anti-mouse $\operatorname{IgG}$ ) at a dilution of 1:100 was added to the sections, which were then incubated at $37^{\circ} \mathrm{C}$ for another $1 \mathrm{~h}$. Factor VIII staining was performed according to the instructions provided with the factor VIII immunochemistry staining kit (Beijing TCT Medical Technology, Beijing, China). For CD68 and CD3 staining, the two antibodies and SP staining kit were purchased from Zhongshanjingqiao Biotechnical Co. Ltd (Beijing, China). The sample embedded in paraffin was deparaffinized and hydrated, followed by SP staining according to the instructions provided with the staining kit, and PBS was used as a negative control.

PCR detection. For the detection of the porcine endogenous retrovirus, primers were designed according to the porcine endogenous retrovirus gag protein sequence (GeneBank accession no. AY265811.1). The forward primer was 5'-GCGACCCACGCAGTTGCATA-3' and the reverse primer was 5'-CAGTTCCT TGCCCAGTGTCCTT-3'. The length of the target fragment was $662 \mathrm{bp}$. The PCR conditions were: $95^{\circ} \mathrm{C}$ for $1 \mathrm{~min}$, annealing at $55^{\circ} \mathrm{C}$ for $1 \mathrm{~min}$ and extension at $72^{\circ} \mathrm{C}$ for $1 \mathrm{~min}$. The PCR product $(5 \mu \mathrm{l})$ was analyzed on a $15 \mathrm{~g} / \mathrm{l}$ agarose gel and visualized by ethidium bromide staining with UV illumination.

Measurement of mechanical properties. The puncture stress (PT) was evaluated and tensile stress determined by stretching the tissue at a rate of $1 \mathrm{~mm} / \mathrm{min}$ using a texture analyzer with a $500 \mathrm{~N}$ load cell.

Aorta grafting. The pig was subjected to intravenous-inhalation anesthesia prior to surgery. The graft was implanted into the descending aorta. The incision was performed at the fourth intercostal space. Subsequently, two intercostal aortas were ligatured. The blood was blocked between the proximal and distal ends of the descending aorta, and a section of descending aorta was removed, $\sim 2 \mathrm{~cm}$ in length. The reconstituted aorta was transplanted by continuous suturing. Following the exhaustion of air, the hemostatic forceps were released. The wound was then staunched and the thorax and lung were washed. Two months after the operation, the implanted blood vessel was dissected out using a similar method to that previously described and the pig was sacrificed.

Statistical analyses. Data are presented as the mean \pm SD. Statistical analysis was performed using SPSS 11.0.0, 2001 (SPSS Inc., Chicago, IL, USA). The Student's t-test was performed for the analysis of differences. $\mathrm{P}<0.05$ was considered to indicate a statistically significant result.

\section{Results}

Histological observation. We histologically examined the reconstituted aorta treated by the vacuum freeze-drying and radiation method. Compared with the normal aorta which had a regular cell arrangement and well structured intima, the graft exhibited numerous small holes with a fundamentally complete media structure and loosely structured spandex fibres, but lacked intima structure (Fig. 1).

We also examined the immunogenicity of the reconstituted aorta. A comparison of the signal strengths of the MHC II-positive cells in the normal $(0.2984 \pm 0.0121)$ and reconstituted aorta $(0.0748 \pm 0.0087)$ revealed that the reconstituted graft had significantly lower immunogenicity than normal aorta $(\mathrm{P}<0.01$; Fig. 2$)$.

To investigate the endogenous retrovirus deactivating effect of the vacuum freeze-drying and radiation method, we analyzed the samples by PCR. Pre-viral DNA was undetectable in the vacuum freeze-drying and radiation treatment 

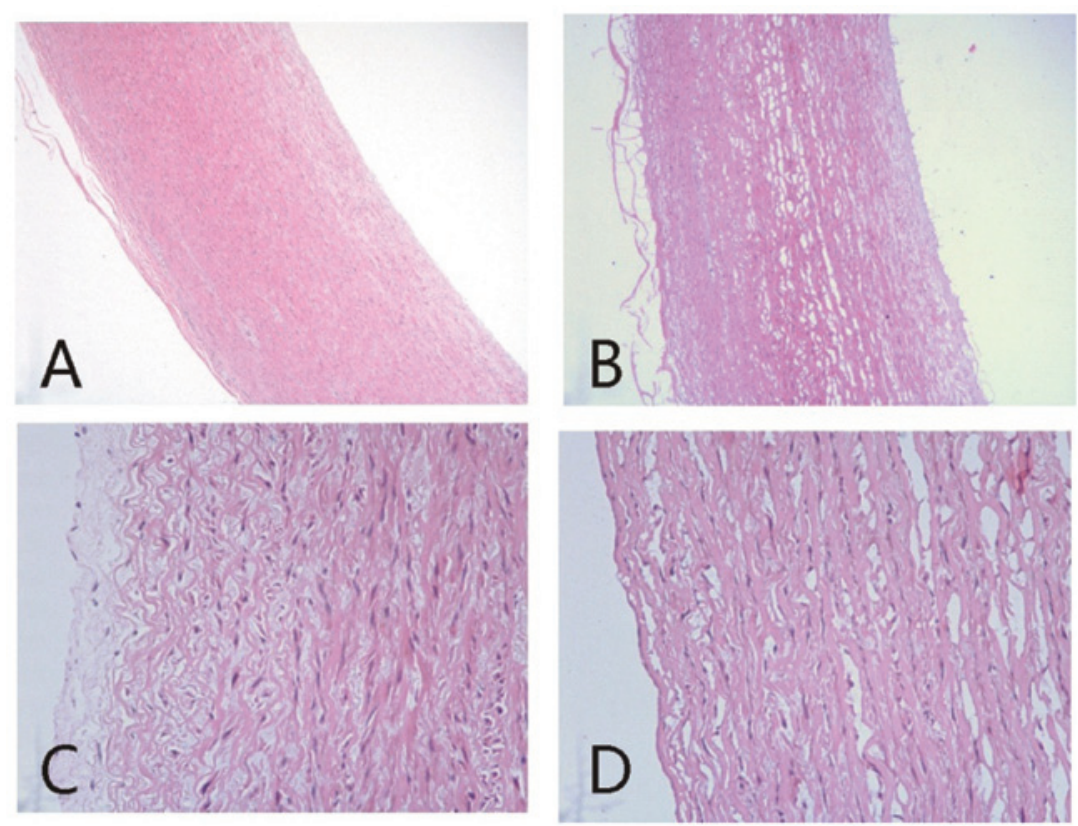

Figure 1. Longitudinal sections of aorta stained with hematoxylin and eosin (H\&E). Normal aorta at magnifications of (A) x400 and (C) x200. The reconstituted aorta treated by vacuum freeze-drying and radiation at magnifications of (B) x400 and (D) x200.
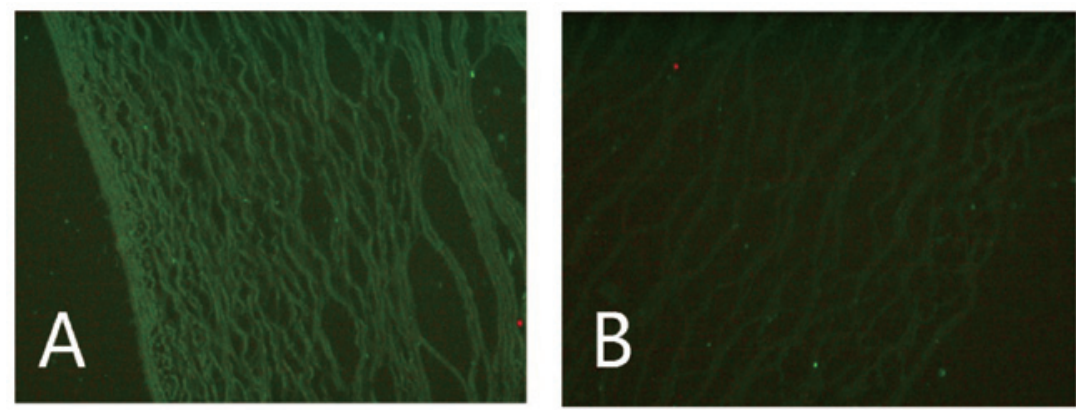

Figure 2. Longitudinal sections of aorta revealed the signals of immunoreactive MHC II-positive cells. (A) Normal aorta and (B) reconstituted aorta, x200.

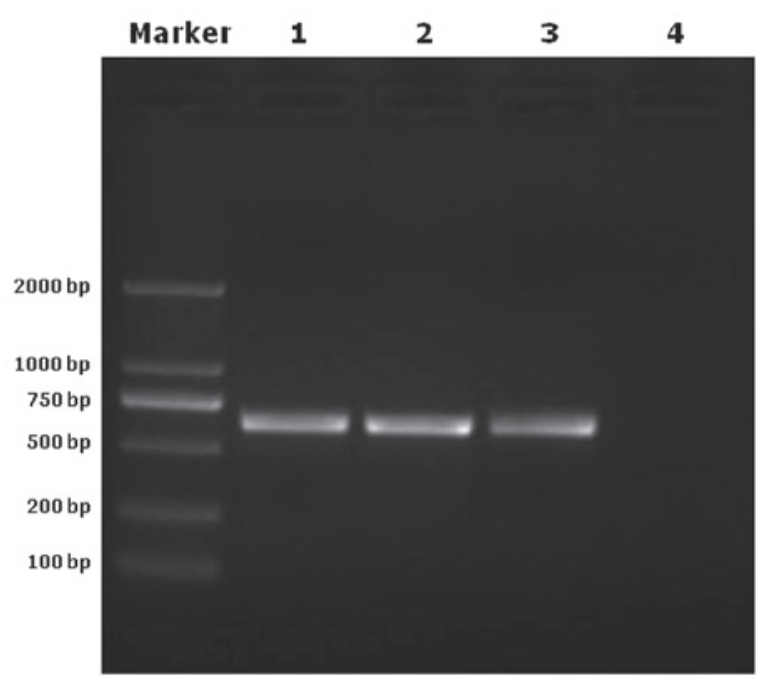

Figure 3. Detection of porcine endogenous retrovirus. The band for the virus was revealed in (1) the porcine endogenous retrovirus $\mathrm{C}$ genome, (2) the normal aorta genome and (3) the genome of the reconstituted aorta treated by vacuum freeze-drying. (4) No band was detected in the genome of the reconstituted aorta treated by vacuum freeze-drying and radiation. M, marker.

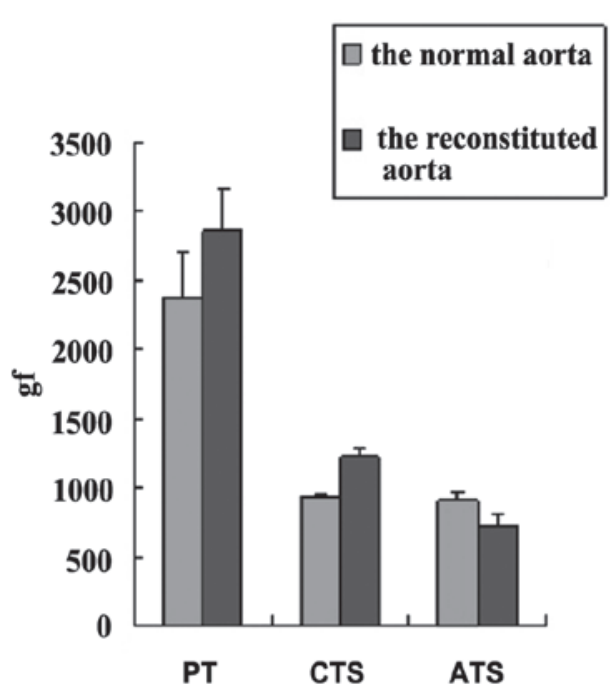

Figure 4. Mechanical properties of the normal aorta and the reconstituted aorta treated by vacuum freeze-drying and radiation. PT, puncture stress; CTS, the maximum circumferential tensile stress; ATS, the maximum axial tensile stress; gf, gram-force. 

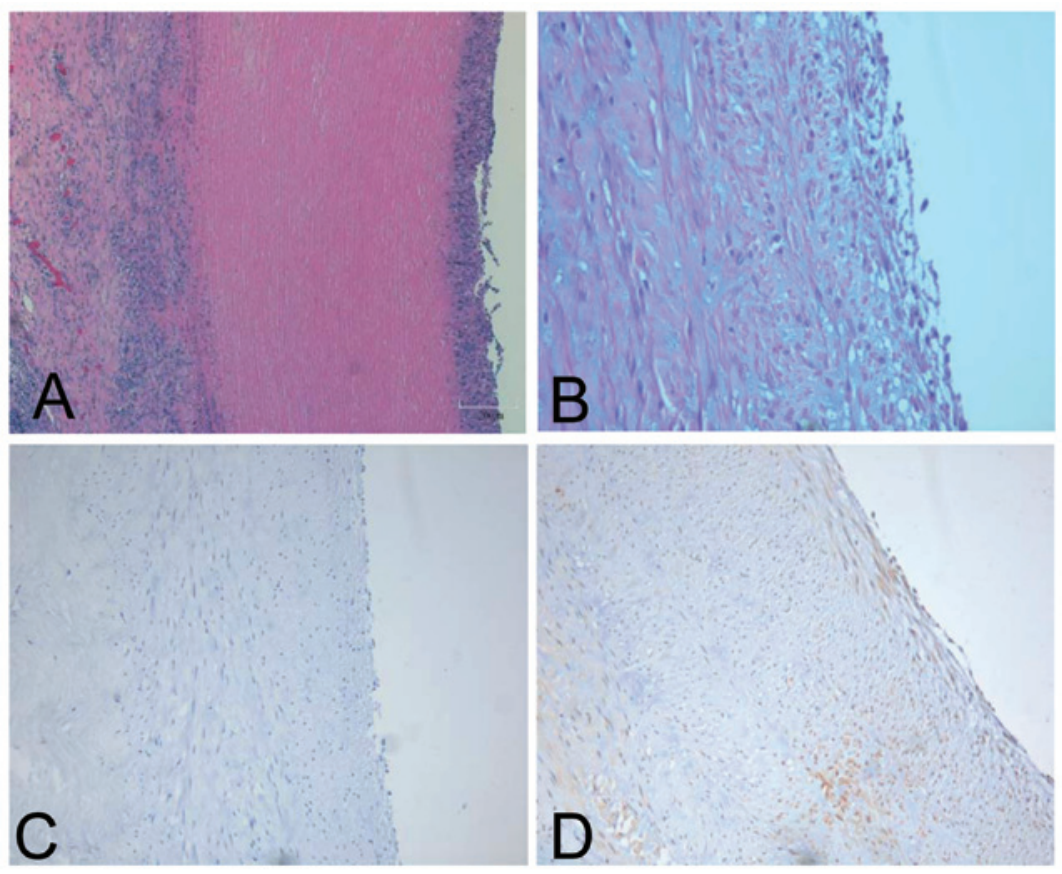

Figure 5. Staining of the aortal graft two months after surgery. (A) Hematoxylin and eosin (H\&E) staining, and immunohistochemical staining for detection of (B) factor VIII, (C) CD68 and (D) CD3 in the graft.

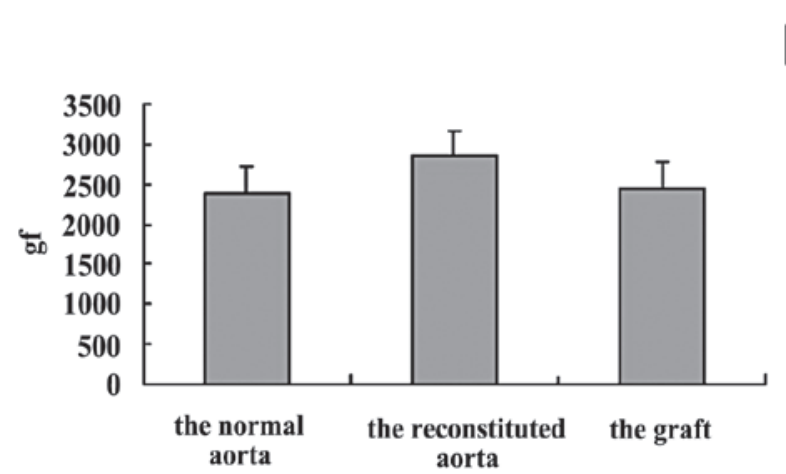

Figure 6. Measurement of puncture stress (PT) in the normal aorta, the reconstituted aorta treated by vacuum freeze-drying and radiation and the graft two months after surgery. gf, gram-force.

group, but was detected in the normal aorta and vacuum freeze drying treatment groups and in the porcine endogenous retrovirus $\mathrm{C}$ genome which served as a positive control (Fig. 3).

Next, we measured the mechanical properties of the reconstituted aorta. Compared with normal aorta, the maximum axial tensile stress (ATS) decreased by $20 \%$, the maximum circumferential tensile stress (CTS) increased by $30 \%$ and the maximum PT decreased by $20 \%$ (Fig. 4).

To investigate the validity of the vacuum freeze-drying and radiation method, aorta grafting was carried out. After the surgery, without using any drugs, abnormal behavior and disease were not detected. Two months after the operation, when the grafts were dissected out, no particular abnormalities were observed. Histologically, clear intima, media and adventitia had formed, endothelial cells had almost covered the inner wall of vessel, and a great number of host cells had migrated into the intima and adventitia in which a small number of inflammatory cells also had migrated (Fig. 5A). Factor VIII staining was performed to distinctly visualize the well-developing host endothelialization (Fig. 5B). Staining for CD68 (a marker for macrophages) and CD3 (a marker for $\mathrm{T}$ lymphocytes) was performed to measure the inflammatory reaction level. The CD68 and CD3 positive lymphocytes were present at very low levels within the adventitia, indicating that the graft lacked immunogenicity (Fig. 5C and D). The PT of the grafts was similar to that of normal aortas (Fig. 6).

\section{Discussion}

The antigenicity of the blood vessel exists in the endothelial cells which are the first point of contact between the recipient and the donor tissue and the first to be recognized by the immune system $(16,17)$. It has been reported that certain parts of the MHC antigenic determinant may be blocked by lyophilization treatment, reducing immunogenicity (18). A murine grafting study revealed that the levels of MHC I and MHC II antigens decreased by $70 \%$ following lyophilization (19). The results of the current study reveal that the vacuum freeze-drying and radiation treatment reduced the MHC II level by $75 \%$. Furthermore, two months after the grafting procedure, only a small number of CD68 or CD3-positive cells were visualized, revealing a low level of inflammatory reaction. This indicates that the vacuum freeze-drying and radiation method may serve as an effective tool for reducing immunogenicity.

It is not clear whether that the intima was required in grafting. In a previous study, the intima of the human heart valve has been demonstrated to be unnecessary in allografts (20). Our study demonstrated that although the intima could not be identified in the reconstituted aorta, two months after the grafting the intima had re-endothelialized. 
Although the reconstituted aorta was in a loose condition, and its mechanical properties had altered, two months after grafting the cells of the graft had rearranged; new intima, media and adventitia had formed and endothelial cells had almost covered the inner wall of the vessel. The structure had nearly returned to that of normal aorta. Moreover, the PT of the graft had already returned to a normal level.

During the two months of the experiment, abnormal behavior and diseases were not detected. No marked size change was observed by the unaided eye when the graft was withdrawn. Hence, the reconstituted aorta may meet the requirements for grafting.

The effects of thermal stress on blood vessels during cryopreservation have already been carefully studied (21-23). Since a fast cooling rate generates higher thermal stresses, the cooling rate greatly affects the thermal stress in the blood vessel during the cooling process. Therefore, a low rate cooling process is required during cryopreservation. In this study, we developed a new cooling process suitable for vessel grafts.

Decades ago, the porcine aorta was first utilized as a replacement for human aorta, but the immunogenicity of the graft and the presence of the porcine endogenous virus limited its clinical application $(24,25)$. The porcine endogenous retrovirus has been reported to have the ability to infect various human cell lines (26), therefore our study aimed to provide a new perspective on xenografts.

In conclusion, we have introduced a vacuum freeze-drying and radiation method for the pre-grafting treatment of porcine aorta, aimed at reducing immunogenicity, preserving vessel structure and mechanical properties, elevating histocompatibility and removing pathogenic microorganisms. In further studies, we propose to combine a decellularizing technique with the vacuum freeze-drying and radiation method to remove the cell debris which may cause calcification in xenografts (27).

\section{Acknowledgements}

This study was financially supported by the Shanghai Municipal Education Commission Innovation Project (no. 2012zz101) and Shanghai Jiaotong University Academic Project (no. YZ1012).

\section{References}

1. Hua ZZ, Xu HY, Zhou GY, Liu JF, Huang HM and Ding WX: Analyses of thermal stress and fracture during cryopreservation of blood vessel. Sci China Ser E-Tech Sci 31: 123-127, 2001.

2. Dahl SL, Koh J, Prabhakar V and Niklason LE: Decellularized native and engineered arterial scaffolds for transplantation. Cell Transplant 12: 659-666, 2003.

3. Gui L, Muto A, Chan SA, Breuer CK and Niklason LE: Development of decellularized human umbilical arteries as small-diameter vascular grafts. Tissue Eng Part A 15: 2665-2676, 2009.

4. Frota AC, Lima Filho AA, Dias AB, Lourenço AC, Antecka E and Burnier MN Jr: Freeze-drying as an alternative method of human sclera preservation. Arq Bras Oftalmol 71: 137-141, 2008.

5. Gherardini G, Haegerstrand A, Matarasso A, Gurlek A, Evans GR and Lundeberg T: Cell adhesion and short-term patency in human endothelium preseeded $1.5-\mathrm{mm}$ polytetrafluoroethylene vascular grafts: an experimental study. Plast Reconstr Surg 99: 472-478, 1997.
6. Weinberg CB and Bell E: A blood vessel model constructed from collagen and cultured vascular cells. Science 231: 397-400, 1986.

7. Matsuda T, Kitamura T, Iwata H, Takano H and Akutsu T: A hybrid artificial vascular graft based upon an organ reconstruction model. Significance and design criteria of an artificial basement membrane. ASAIO Trans 34: 640-643, 1988.

8. Ratcliffe A: Tissue engineering of vascular grafts. Matrix Biol 19: 353-357, 2000.

9. Guymer RH and Mandel TE: A comparison of corneal, pancreas, and skin grafts in mice. A study of the determinants of tissue immunogenicity. Transplantation 57: 1251-1262, 1994.

10. Umscheid T and Stelter WJ: Time-related alterations in shape, position, and structure of self-expanding, modular aortic stentgrafts: a 4-year single-center follow-up. J Endovasc Surg 6: 17-32, 1999.

11. Hiles MC, Badylak SF, Lantz GC, Kokini K, Geddes LA and Morff RJ: Mechanical properties of xenogeneic small-intestinal submucosa when used as an aortic graft in the dog. J Biomed Mater Res 29: 883-891, 1995.

12. Bacon LD and Motta J: Skin-graft histocompatibility within Regional Poultry Research Laboratory inbred chicken lines. Poult Sci 61: 218-220, 1982.

13. Chang $\mathrm{C}$ and Zhang J: The analysis of relationship between fetal stress and blood dynamics in fetal vessels and placental bed vessels. Zhonghua Fu Chan Ke Za Zhi 31: 15-17, 1996 (In Chinese).

14. Rose AG, Forman R and Bowen RM: Calcification of glutaraldehyde-fixed porcine xenograft. Thorax 33: 111-114, 1978.

15. Yin M, Liu MF, Cao Q, Wu JQ and Tao LR: Mechanical properties of porcine aorta after vacuum freeze-drying processes. J Clin Rehab Tissue Eng Res 14: 9539-9544, 2010.

16. Timaran $\mathrm{CH}$, Stevens SL, Freeman $\mathrm{MB}$ and Goldman $\mathrm{MH}$ : Infrainguinal bypass grafting using lyophilized saphenous vein allografts for limb salvage. Cardiovasc Surg 10: 315-319, 2002.

17. Cooper DK: Clinical survey of heart transplantation between ABO blood group-incompatible recipients and donors. J Heart Transplant 9: 376-381, 1990.

18. Vesely I, Gonzalez-Lavin L, Graf D and Boughner D: Mechanical testing of cryopreserved aortic allografts. Comparison with xenografts and fresh tissue. J Thorac Cardiovasc Surg 99: 119-123, 1990.

19. Cattral MS, Warnock GL, Kneteman NM, Halloran PF and Rajotte RV: The effect of cryopreservation on the survival and MHC antigen expression of murine islet allografts. Transplantation 55: 159-163, 1993.

20. Gavin JB, Herdson PB, Monro JL and Barratt-Boyes BG: Pathology of antibiotic-treated human heart valve allografts. Thorax 28: 473-481, 1973.

21. Zhao G, Liu ZF, Zhang AL, Zhang HF and Cheng SX: Theoretical analyses of thermal stress of blood vessel during cryopreservation. Cryo Letters 26: 239-250, 2005.

22. Pegg DE, Wusteman MC and Boylan S: Fractures in cryopreserved elastic arteries. Cryobiology 34: 183-192, 1997.

23. Bujan J, Pascual G, Lopez R, et al: Gradual thawing improves the preservation of cryopreserved arteries. Cryobiology 42: 256-265, 2001.

24. Zuhdi N, Hawley W, Voehl V, Hancock W, Carey J and Greer A: Porcine aortic valves as replacements for human heart valves. Ann Thorac Surg 17: 479-491, 1974.

25. Sawyer PN: Experimental observations on antithrombotic biophysical phenomena in normal and atherosclerotic human aorta and porcine aortic grafts. Ann NY Acad Sci 149: 628-642, 1968.

26. Leyh RG, Wilhelmi M, Walles T, et al: Acellularized porcine heart valve scaffolds for heart valve tissue engineering and the risk of cross-species transmission of porcine endogenous retrovirus. J Thorac Cardiovasc Surg 126: 1000-1004, 2003.

27. Rieder E, Kasimir MT, Silberhumer G, et al: Decellularization protocols of porcine heart valves differ importantly in efficiency of cell removal and susceptibility of the matrix to recellularization with human vascular cells. J Thorac Cardiovasc Surg 127: 399-405, 2004. 